\title{
Power of QTL mapping experiments in commercial Atlantic salmon populations, exploiting linkage and linkage disequilibrium and effect of limited recombination in males
}

\author{
BJ Hayes ${ }^{1,2}$, A Gjuvsland ${ }^{1}$ and S Omholt ${ }^{1}$ \\ ${ }^{1}$ AKVAFORSK, Institute for Aquaculture Research, PO 5010, 1432 Ås, Norway; ${ }^{2}$ CIGENE (Centre for Integrative Genetics), \\ Agricultural University of Norway, PO.Box 5003 N-1432 Ås, Norway
}

\begin{abstract}
Whereas detection and positioning of genes that affect quantitative traits (quantitative trait loci (QTL)) using linkage mapping uses only information from recombinants in the genotyped generations, linkage disequilibrium (LD) mapping uses historical recombinants. Thus, whereas linkage mapping requires large family sizes to detect and accurately position QTL, LD mapping is more dependant on the number of families sampled from the population. In commercial Atlantic salmon breeding programmes, only a small number of individuals per family are routinely phenotyped for traits such as disease resistance and meat colour. In this paper, we assess the power and accuracy of combined linkage disequilibrium linkage analysis (LDLA) to detect QTL in the commercial population using simulation. When 15 half-sib sire families (each sire mated to 30 dams, each dam with 10 progeny) were sampled from the population for genotyping,
\end{abstract}

we were able to detect a QTL explaining $10 \%$ of the phenotypic variance in $85 \%$ of replicates and position this QTL within $3 \mathrm{cM}$ of the true position in $70 \%$ of replicates. When recombination was absent in males, a feature of the salmon genome, power to detect QTL increased; however, the accuracy of positioning the QTL was decreased. By increasing the number of sire families sampled from the population to be genotyped to 30 , we were able to increase both the proportion of QTL detected and correctly positioned (even with no recombination in males). QTL with much smaller effect could also be detected. The results suggest that even with the existing recording structure in commercial salmon breeding programmes, there is considerable power to detect and accurately position QTL using LDLA.

Heredity (2006) 97, 19-26. doi:10.1038/sj.hdy.6800827; published online 10 May 2006

Keywords: QTL; linkage disequilibrium mapping; commercial salmon populations

\section{Introduction}

Although traditional selective breeding has led to rapid gains in economically important traits in Atlantic salmon (eg Gjedrem, 1997), faster gains could be achieved if the genes affecting these traits (termed quantitative trait loci (QTL)) could be identified. This information could then be used in marker-assisted selection (MAS). The improvement from MAS is expected to be particularly large for traits that are difficult to select for (Meuwissen and Goddard, 1996). One example is meat colour, where in salmon selection programmes, the trait is only measured on relatives of the breeding candidates.

A key question is whether QTL for economically important traits can be detected in currently available commercial Atlantic salmon populations, and within the existing phenotype recording structure (Figure 1). In this structure, a few progeny from a large number of families are evaluated for traits such as meat quality and disease resistance. Many QTL detection experiments use linkage mapping in half-sib or full-sib families (eg Jackson

Correspondence: BJ Hayes, AKVAFORSK, Institute for Aquaculture Research, PO 5010, 1432 As, Norway.

E-mail:ben.hayes@akvaforsk.no

Received 29 July 2005; accepted 6 March 2006; published online 10 May 2006 et al, 1998; Sakamoto et al, 1999). In linkage mapping, the difference in the average phenotypic value between progeny within half- or full-sib families receiving alternate parental marker alleles is calculated. For successful detection of QTL, the size of the families must be large enough so that difference between progeny groups receiving alternate marker alleles is significantly greater than the effects of other genes and environmental effects (eg Haley and Knott, 1992). As an example, to detect a QTL with a gene substitution effect of 0.4 environmental standard deviations and allele frequencies of 0.5 with $95 \%$ probability, four half-sib families of 250 progeny each would be required (Martinez et al, 2002). Full-sib family groups of this size are much larger than are currently phenotyped for any one trait in the commercial breeding companies. An alternative to linkage analysis (LA) is to use linkage disequilibrium and linkage analysis (LDLA) information simultaneously (eg Meuwissen et al, 2002; Blott et al, 2003; Fan et al, 2005). Linkage disequilibrium (LD) between a marker and a QTL implies that there is an association between an allele at the marker locus and an allele at the QTL across the entire population, and not just within families. In pure LD mapping, no family structure is required. The LDLA strategy uses both the linkage and LD information to determine the probability of a QTL being present at a 


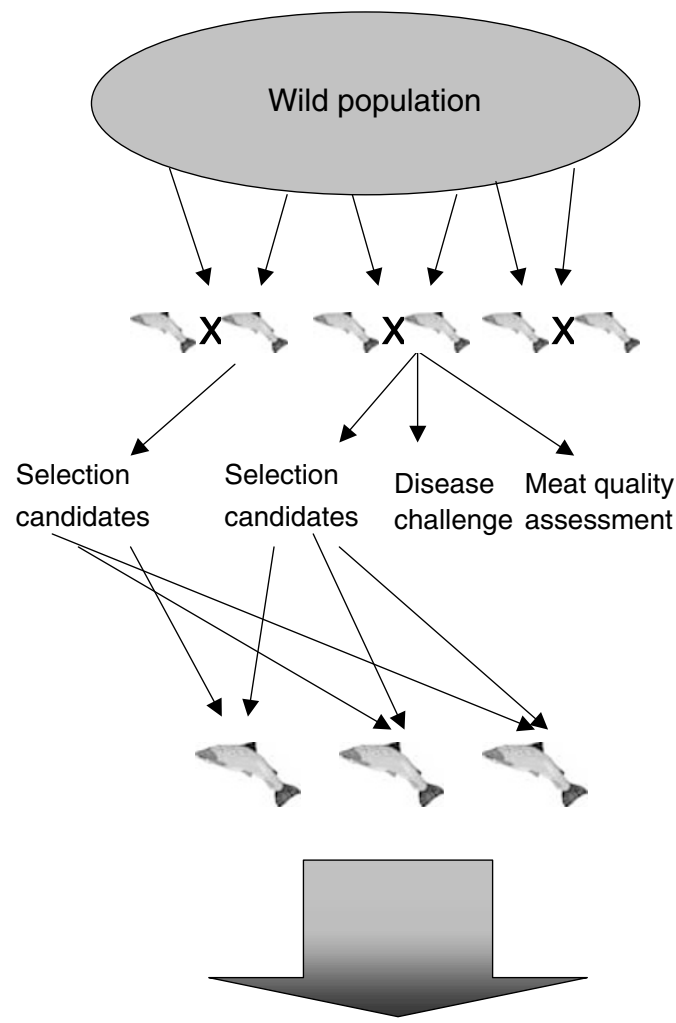
1. Sample males and females at random from wild population

2. Mate to create 300 (full sib) family lines

3. Each family split into three: selection candidates, disease challenge animals (10 per family) and meat quality assessment animals (10 per family). Growth recorded on all animals

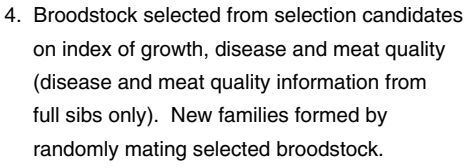
on index of growth, disease and meat quality (disease and meat quality information from full sibs only). New families formed by randomly mating selected broodstock.

5. Steps 3 to 4 repeated for each generation of breeding (7 generations of breeding to date)

Figure 1 Structure of Norwegian Atlantic salmon breeding programme for a single breeding company.

particular site in the genome. As the extent of across population LD is typically much less than within families (eg Farnir et al, 2000; McRae et al, 2001), the LDLA approach also requires a denser marker map in order to gain information from across population LD. In livestock, such maps are quickly becoming a reality (eg ftp:/ /ftp.hgsc.bcm.tmc.edu/pub/data/Btaurus/snp/ Btau20040927/bovine-snp.txt), and many markers are now available for both Atlantic salmon and catfish (He et al, 2003; Hayes et al, 2004). Additionally, the development of new genotyping technologies is drastically reducing the cost of genotyping, particularly for single nucleotide polymorphism (SNP) markers (eg Bell et al, 2002). In this study, we evaluate the power of both LDLA and LA to detect and position QTL using the existing commercial population and phenotype recording structure. We also investigate the effect of a novel feature of the salmon genome, that there is little or no recombination among some of the male chromosomes during meiosis (Moen et al, 2004a, b), on the power of QTL detection. Finally, a strategy that aims to reduce the cost of genotyping needed to detect QTL with LDLA was evaluated.

\section{Methods}

\section{Simulation}

A base population of Atlantic salmon of size 100 individuals was created by computer simulation. The validity of effective population size 100 is considered in the discussion. Each animal in the population had two chromosome segments (one from its mother and one from its father). On a chromosome segment, there were 10 markers and one QTL locus, in the centre of the segment, for example, M_M_M_M_MQM_M_M_M_M. The length of the chromosome segment was $10 \mathrm{cM}$, for example, a 1/10 chance of recombination during any meiosis. During a meiosis, the probability of mutation at a marker or QTL was 0.0017 (a justification for this mutation rate is given in the Results section). If a locus was mutated, a new allele was added at that locus. Random mating was carried out for 1000 generations to produce a population in mutation-drift balance. Ten generations of exponential growth of this population followed such that there were 600 individuals in generation 1010 .

In generation 1010, 150 sires were chosen and 300 dams. Each sire was mated to two dams to produce 10 offspring per mating (total number of progeny 3000). This design is similar to that used by the two salmon breeding companies in Norway today, with 300 families and approximately 10 individuals per family evaluated for meat colour and disease resistance. The population was simulated both with and without recombination in males.

In the final generation, the effects of the QTL alleles were sampled from a normal distribution $N \sim(0,1)$. In our simulations, $2-5$ alleles were typically segregating at the QTL. To generate a phenotypic record for each of the 3000 progeny, the effects of the two QTL alleles a progeny carried were summed, and a random environmental component sampled from a normal distribution $N \sim(0,1)$. Sire, dam, and Mendelian sampling terms were also added, such that the proportion of the total phenotypic variance explained by the QTL 
was either $0.1,0.05$, or 0.025 , and the heritability of the trait was 0.4 .

To assess the effect of a larger effective population size in the population before the formation of the breeding population, an additional simulation was performed where a base population of size 1000 individuals was simulated for 6000 generations, with mutation rate at the markers and QTL of 0.00017. This was followed by 10 generations of effective population size of 100 (with the aim of simulating the effect of sampling the breeding population from the wider population). Then in generation 6011, 150 sires and 300 dams were selected as described above to produce 300 full-sib families of 10 progeny per family.

\section{Genotyping strategies}

Either 15 or 30 half-sib families (with 20 or 10 progeny per half-sib family, respectively) were selected for genotyping from the 150 half-sib families in the population. In the first genotyping strategy, strategy RAND, this selection was made at random. In the second strategy, the 15 half-sib families with the maximum within half-sib family variance (calculated from the phenotypic records) were selected for genotyping (strategy MAXV). The aim of this strategy was to try and select only those families from sires heterozygous at the QTL.

We also assessed (only for the situation with no recombination in males) the effect of increasing the size on the mapping population on the power to detect and position QTL of different sizes. The number of half-sib families selected for genotyping was increased to 30 (20 progeny per half-sib family).

\section{QTL analysis}

Two methods of analysis were used: LA and combined LDLA. For the LA, a two-step variance component method (eg George et al, 2000) was used. The two steps were as follows:

1. For each QTL position on the chromosome segment, calculate the (co)variance matrix associated with the QTL. This matrix is also called the G or identical by descent (IBD) matrix, and has elements $i j=$ Prob(QTL alleles $i$ and $j$ are IBD). We used the LOKI package (Heath, 1997), which uses a Markov chain MonteCarlo approach to estimate the IBD matrix.

2. For each position considered in step 1, construct a linear model to estimate QTL variance and other parameters, test for the presence of a QTL. The linear model was $Y=\mu+u+Z v+e$, where $Y$ is a vector of observed phenotypes, $\mu$ the overall mean, $u$ the vector of random polygenic effects, $v$ the vector of random QTL effects, $e$ the residual vector, and $Z$ the design matrix relating the QTL allele to the phenotype. The random effects $u, v$, and $e$ are assumed to be distributed as follows: $u \sim N\left(0, \sigma_{u}^{2} A\right), v \sim N\left(0, \sigma_{v}^{2} G\right)$, $e \sim N\left(0, \sigma_{e}^{2} I\right)$, where $\sigma_{u}^{2}, \sigma_{v}^{2}$, and $\sigma_{e}^{2}$ are the polygenic variance, the additive QTL variance of one allele, and the residual variance, respectively. $A$ is the standard additive genetic relationship matrix and $G$ is the IBD matrix described above. Parameters $\sigma_{u}^{2}, \sigma_{v}^{2}$, and $\sigma_{e}^{2}$ were estimated using ASReml statistical package (Gilmour et al, 2002), which also calculated the likelihood of the above model.
Table 1 The matrix of IBD coefficents

\begin{tabular}{lccc}
\hline & $\begin{array}{c}\text { Sire } \\
\text { haplotypes }\end{array}$ & $\begin{array}{c}\text { Dam } \\
\text { haplotypes }\end{array}$ & $\begin{array}{c}\text { Progeny } \\
\text { haplotypes }\end{array}$ \\
\hline Sire haplotypes & {$[\mathrm{a}]$} & {$[\mathrm{a}]$} & {$[\mathrm{b}]$} \\
Dam haplotypes & {$[\mathrm{a}]$} & {$[\mathrm{a}]$} & {$[\mathrm{b}]$} \\
Progeny haplotypes & {$[\mathrm{b}]$} & {$[\mathrm{b}]$} & {$[\mathrm{b}]$} \\
\hline
\end{tabular}

IBD, identical by descent; LD, linkage disequilibrium.

The IBD matrix has a row for each sire haplotype, a row for each dam haplotype, and a row for each of the progeny haplotypes. The IBD coefficents among the sire and dam haplotypes (a blocks) are generated using LD only, using the method of Meuwissen and Goddard (2001). IBD coefficents between sire and progeny and dam and progeny haplotypes (b blocks) are based on linkage and are generated following Meuwissen et al (2002).

For the LDLA, a two-step procedure was also used. In the identical by descent (IBD) matrix, the $(i, j)$ element is the probability that the QTL alleles in gametes $i$ and $j$ are IBD. The IBD matrix was calculated using the method described by Meuwissen et al (2002). Marker haplotypes (marker alleles that occur on either the maternal or paternal chromosome) of all the parents and the progeny were used for the prediction of IBD probabilities, which formed the IBD matrix in Table 2. The probability of IBD among the base QTL alleles (block [a] in Table 1) is based on LD, whereas IBD probabilities in block [b] are based on linkage, that is, tracing chromosome segments from sires and dams to progeny.

The second step of the analysis was the estimation of QTL parameters, and was carried out using ASREML as described above.

For both LA and LDLA, the IBD probabilities, the likelihood of the model, and the variances were assessed for a putative QTL in the middle of each marker bracket. The likelihood ratio (LRT) was calculated as twice the difference in log-likelihoods between the model with the QTL and the model without the QTL. A QTL was detected if this value exceeded $\chi_{0.1}^{2}=2.71$. The most likely bracket containing the QTL was the one with the highest LRT, at which the QTL variance was estimated. The QTL was considered to be correctly positioned if the estimated position was \pm one bracket from the true position.

There were 25 replicate populations of each mapping design.

\section{Results}

\section{Simulated populations}

To determine if our simulation model was producing populations in mutation-drift balance, we investigated the average heterozygosities of the markers across generations, and the amount of LD between the markers. Under a mutation-drift model with no selection, the equilibrium value for heterozygosity should be

$$
H=\frac{4 N u}{4 N u+1}
$$

(Kimura and Crow, 1964), where $N$ is the effective population size and $u$ is the mutation rate per locus per gamete. In our case, $N$ was 100 and $u$ was 0.0017 , so the expected heterozygosity per marker would be 

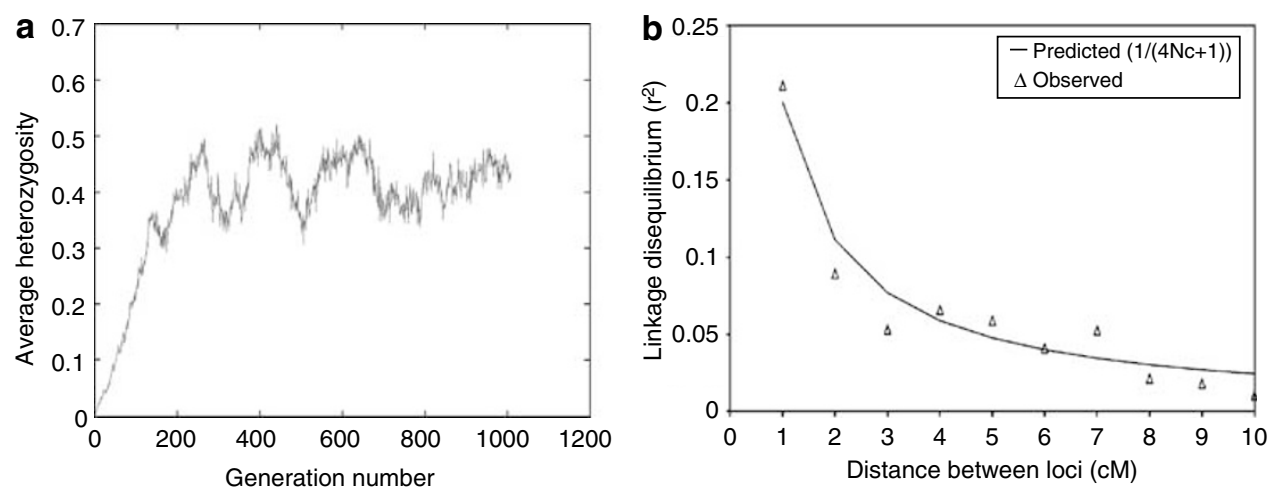

Figure 2 (a) Average marker heterozygosity across generations from a single simulation and (b) pairwise LD ( $r 2)$ between markers in generation 1000 from a single replicate.

0.40. The expected heterozygosity agreed well with the average heterozygosity across marker loci observed in the simulations (Figure 2a), following an initial period (generations $0-400$ ) of accumulation of mutations. The mutation rate was actually chosen to give a heterozygosity of approximately 0.4, as although a heterozygosity of 0.4 is somewhat lower than is typically observed with microsatellite markers (eg Withler et al, 2005), it is similar to that observed for SNPs in SNP panels (Hayes et al, 2004). The heterozygosity of the QTL was also approximately 0.4 , which would be equivalent to a bi-allelic QTL with a rare allele frequency of 0.28 . This is similar to the rare allele frequency of QTL detected in some salmon QTL mapping experiments using outbred populations (eg Perry et al, 2001). If the heterozygosity of the QTL were lower than this, the power of QTL mapping in the results below would be reduced, as there would be less phenotypic observations on one of the QTL alleles. However, if the heterozygosity were increased, power would also be increased.

When the population size was increased to 1000, the mutation rate at QTL and markers was reduced to 0.00017 , in order to maintain a heterozygosity of 0.4 . The heterozygosity was maintained at 0.4 (results not shown) so that the differences in the power of QTL mapping in this population and the population of smaller $N$ depended only on the amount of LD, and not marker or QTL heterozygosity.

Under a finite population size model, the amount of LD between two loci separated by $c$ Morgans is expected to be $E\left(r^{2}\right)=1 /(4 N c+1)$, where $N$ is the effective population size (Sved 1971). The observed $r^{2}$, calculated using the formula of Hudson (1985), agreed well with the expected result when a single replicate was investigated (Figure 2b). This suggests our simulation model is generating a population with the expected amount of LD.

\section{Power of mapping designs to detect QTL}

It is important to note that owing to computational requirements, only a relatively small number of replicates (25) could be performed for each strategy; so results presented in Tables 2 and 3 are likely to have large standard errors. Despite this, some obvious results were apparent.
Table 2 Proportion of QTL detected with different family structures, different genotyping strategies, and different QTL mapping methodologies

\begin{tabular}{llll}
\hline Design & $\begin{array}{l}\text { Genotyping } \\
\text { strategy }\end{array}$ & Linkage & LDLA \\
\hline $\begin{array}{l}\text { With recombination in males } \\
15 \text { sires, 30 dams, 300 progeny }\end{array}$ & RAND & 0.57 & 0.85 \\
30 sires, 60 dams, 300 progeny & RAND & 0.52 & 0.91 \\
15 sires, 30 dams, 300 progeny & MAXV & 0.87 & 0.96 \\
$\begin{array}{l}\text { With no recombination in males } \\
\begin{array}{l}15 \text { sires, 30 dams, 300 progeny, no } \\
\text { recombination in males }\end{array}\end{array}$ & RAND & 0.64 & 0.75 \\
\hline
\end{tabular}

QTL, quantitative trait loci.

Table 3 Proportion of QTL correctly positioned with different family structures, different genotyping strategies, and different QTL mapping methodologies

\begin{tabular}{|c|c|c|c|}
\hline Design & $\begin{array}{l}\text { Genotyping } \\
\text { strategy }\end{array}$ & Linkage & $L D L A$ \\
\hline \multicolumn{4}{|l|}{ With recombination in males } \\
\hline 15 sires, 30 dams, 300 progeny & RAND & 0.22 & 0.70 \\
\hline 30 sires, 60 dams, 300 progeny & RAND & 0.43 & 0.64 \\
\hline 15 sires, 30 dams, 300 progeny & MAXV & 0.56 & 0.61 \\
\hline \multicolumn{4}{|l|}{ With no recombination in males } \\
\hline $\begin{array}{l}15 \text { sires, } 30 \text { dams, } 300 \text { progeny, } \\
\text { no recombination in males }\end{array}$ & RAND & 0.44 & 0.40 \\
\hline
\end{tabular}

LDLA, linkage disequilibrium linkage analysis; QTL, quantitative trait loci.

In all designs, LDLA detected more QTL than LA alone (Table 2). When genotyping strategy RAND was used, the highest proportion of QTL were detected when 30 sires were mated to 60 dams, that is, when the number of parents sampled from the population was greatest.

The power to detect QTL was increased significantly (in LA) when there was no recombination in males (Table 2 and Figure 3).

Using the MAXV strategy to select which families to genotype substantially increased the power of both LA and LDLA to detect the QTL. 
Power of mapping designs to correctly position QTL

The LDLA gave considerable improvements over LA in the accuracy of positioning the QTL within the $10 \mathrm{cM}$ segment (Table 3). Lack of recombination in males reduced the ability of both methods to accurately position the QTL (Table 3 and Figure 3).

The MAXV strategy showed no superiority over the other strategies in terms of accuracy of positioning the QTL.

\section{Effect of increased past effective population size}

When the effective population size of the base population was increased to 1000 , the amount of LD in the population, especially between loci $1-3 \mathrm{cM}$ apart, fell substantially (compare Figures 4 and $2 b$ ). Note that in Figure 4, the observed data do not fit the prediction of the amount of LD with either $N=1000$ or $N=100$, this is because $N$ is changing over time (from 1000 to 100). The figure shows how the $r^{2}$ quickly approaches the new equilibrium at large distances between markers, but not at small distances. This trend is in agreement with Goddard (1991) and is explained by Hayes et al (2003).

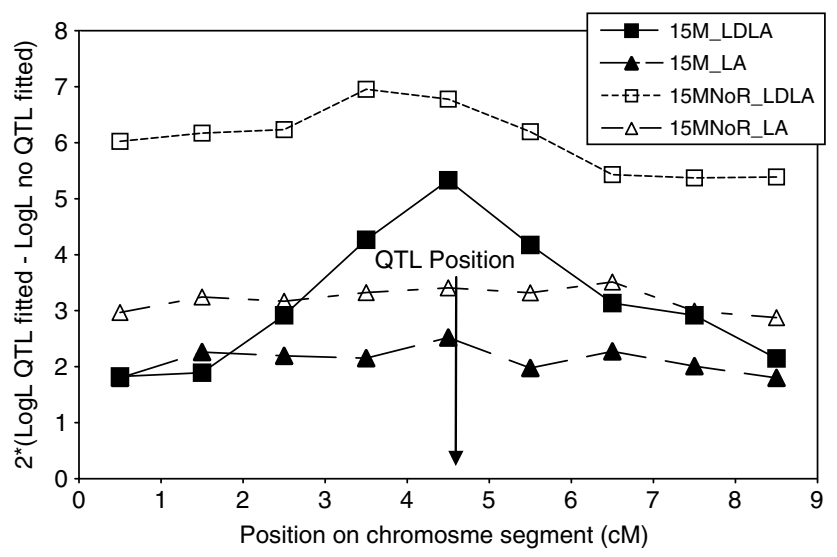

Figure 3 Average likelihood profiles from LA and combined LDLA in simulated Atlantic salmon families with (15M) and without (15MnoR) recombination in males.

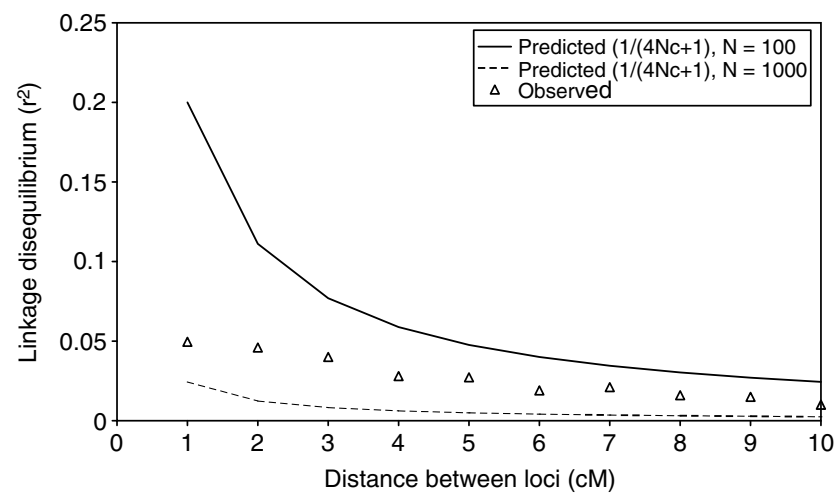

Figure 4 Observed LD $\left(r^{2}\right)$ in population of effective size of 1000 for 6000 generations and 100 for the last 10 generations (average of 25 replicates). Expected LD when effective population size is either 1000 or 100 is also plotted.
In this population (with 15 sires mated to 30 dams and 10 progeny per dam), the proportion of genes detected by the LDLA method was 0.69 , whereas the proportion of genes correctly positioned was 0.63 , which is 0.16 and 0.07 less than the proportion of genes detected and correctly positioned, respectively, when the population size was constant at 100 (Tables 2 and 3). LD is still contributing to the detection and positioning of the QTL; however, with no contribution from LD, the results could be expected to be the same as those from LA alone (proportion of QTL detected 0.65 and proportion of QTL correctly positioned 0.35). As expected, results from LA in this population were very similar to those in Tables 2 and 3.

\section{Effect of increasing the size of mapping population}

Increasing the number of families genotyped substantially increased both the power to detect QTL and the accuracy of positioning the QTL when LDLA was used, Figure 5 (compare results for 15 sires, 30 dams, 300 progeny no recombination in males in Tables 2 and 3 with Figure 3). The power to detect QTL with LA was also improved, but there was no improvement in the accuracy of locating the QTL.

When the MAXV strategy was used to select the 30 half-sib families for genotyping, and with LDLA, we were able to detect a QTL explaining only $2.5 \%$ of the phenotypic variance in $81 \%$ of replicates, and assign this QTL to the correct $3 \mathrm{cM}$ interval in $47 \%$ of cases.
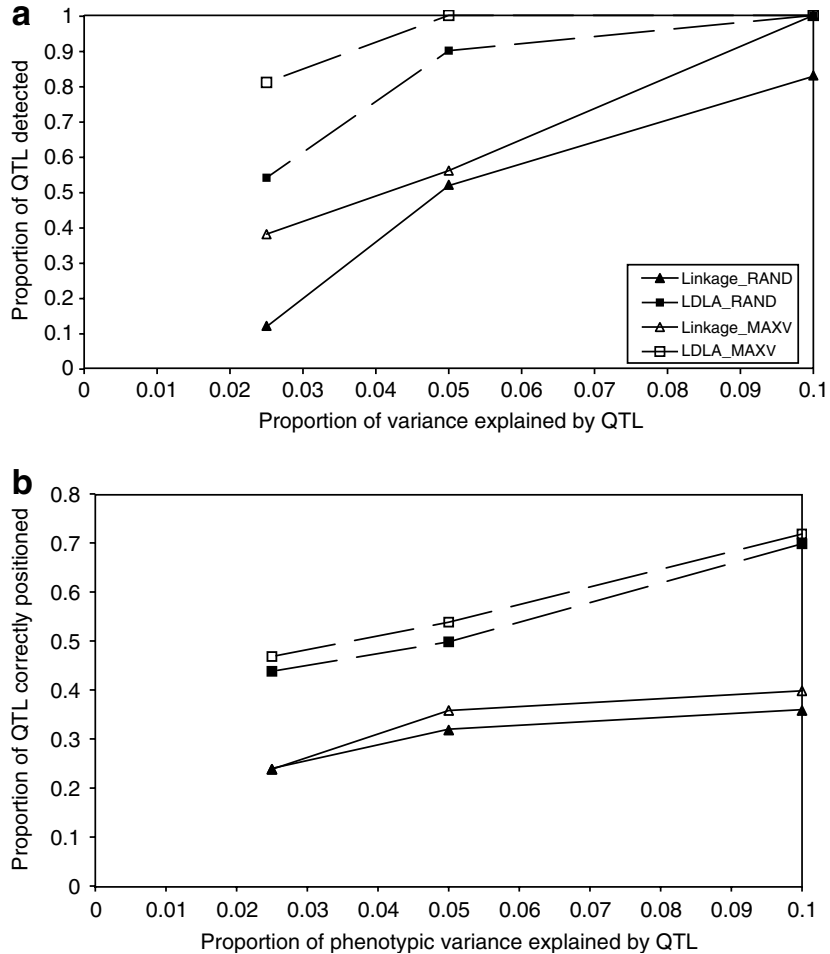

Figure 5 Power of a larger mapping design (30 sires mated to 60 dams in a half-sib design and 10 progeny per dam, no recombination in males) to (a) detect QTL and (b) position QTL within $3 \mathrm{cM}$ of the true QTL position, for QTL explaining different proportions of the phenotypic variance. The 30 half-sib families were randomly selected (RAND) from the breeding population for genotyping, or the half-sib families with the largest within half-sib family variance for the trait (MAXV) were selected. 


\section{Discussion}

To date, all of the QTL mapping experiments carried out in Atlantic salmon (eg Moen et al, 2004a, b; Reid et al, 2005) have been carried out in specially created fullor half-sib families, and using LA. Our results indicate that by using LDLA, QTL of moderate effect (explaining $10 \%$ of the phenotypic variance) can be detected and accurately positioned by sampling families from the current commercial breeding populations, and using phenotypic records from the existing recording scheme. The information from LD or ancestral recombinations contributes substantially both to the success (in terms of probability of detecting QTL) and precision of mapping: up to $39 \%$ more QTL were detected and $48 \%$ more QTL were correctly positioned when LDLA was used compared with LA alone.

The observed increase in the power of LDLA over LA concurs with the results of Lee and van der Werf (2004). They simulated a QTL explaining $25 \%$ of the phenotypic variance segregating in a population of 128 individuals, belonging to between 2 and 64 half-sib families (ie between 64 and 2 individuals per half-sib family). In their study, the use of LD information increased the proportion of replicates in which a QTL was correctly positioned (within a $3 \mathrm{cM}$ bracket) from approximately $30-65 \%$, with a larger advantage of LDLA over LA with more half-sib families in the population.

Reduced or absent recombination for some chromosomes during male gametogenesis in Atlantic salmon has been documented (eg Moen et al, 2004a, b). In both LDLA and LA, lack of recombination in males substantially increased the power to detect QTL, whereas the precision of the QTL mapping was reduced. Lack of recombination in males results in fewer haplotypes, or allelic combinations, being present in the population. In LD mapping, this means that there are more phenotypic observations per haplotype. The estimated differences between haplotypes carrying different QTL alleles will then be estimated more precisely, so QTL are more likely to be detected. However, the power to position QTL is decreased, as the difference in the amount of LD between the QTL and adjacent markers and markers some distance from the QTL will be reduced, compared to a situation where recombination in males occurs. To improve the accuracy of positioning the QTL when there is no recombination in males, more haplotypes must be sampled from the population, that is, progeny from a greater number of families must be genotyped. For example, when the number of sire families genotyped was increased to 30, we were able to detect a QTL explaining $5 \%$ of the phenotypic variance in $100 \%$ of replicates and position this QTL within the correct $3 \mathrm{cM}$ bracket in $54 \%$ of replicates (Figure 3 ).

Exploiting LD in QTL mapping requires a dense marker map. We simulated a chromosome segment with a marker every cM. If there was no prior information on the QTL position, screening the entire genome with this marker density would require approximately 900 markers (based on a map length of $900 \mathrm{cM}$ estimated by Moen et al, 2004a,b). Although this number of SNP markers will be available for Atlantic salmon in the near future (Hayes et al, 2004), an alternative two-stage strategy to genotyping all markers in all fish is possible. This strategy exploits the reduced level of recombina- tion in males. Step 1. Two to three markers from each chromosome are genotyped and the data analysed using LA, to determine which chromosomes harbour QTLs (more markers provide little additional information owing to the limited recombination in males). Step 2. For these chromosomes, genotype markers every cM and analyse the genotype and phenotype data using LDLA.

A second alternative is to exploit the haplotype block structure of LD to reduce the number of markers genotyped. LD occurs because a number of animals in the modern population have inherited the same piece of chromosome from an ancestor who lived perhaps many generations ago. Recombinations will have occurred as the chromosome is passed down the generations, so that perhaps only small chromosome segments are shared by all modern descendants of this ancestor. In these regions, all individuals sharing this small chromosome segment will have the same combination of marker alleles or marker haplotype for the markers in that chromosome segment. Knowledge of the marker haplotypes allows selection of a limited subset of markers that are informative about the remainder of the common markers in a region. These markers are called tag markers, and if the markers are single nucleotide polymorphisms, tag SNPs (Sebastiani et al, 2003). The reduction in the number of SNPs that need to be genotyped depends on the extent of LD and the marker density. In our example, with markers every $\mathrm{cM}$ and effective population size of 100, the reduction in the number of SNPs by using haplotype tags would not be expected to be very large, as the $r^{2}$ between adjacent markers is expected to be $1 /(4 N c+1)=0.2$. In other words, adjacent SNPs are only expected to account for $20 \%$ of the variance of the other marker. But to test this, we used the program BEST (Best Enumeration of SNP Tags; Sebastiani et al, 2003) to select tag SNPs from the 10 markers simulated. The average number of tag SNPs selected in 25 replicates was 8.5 . This would represent only a small reduction in the amount of genotyping required for QTL mapping.

As stated previously, the extent of LD in a population depends on, among other factors, the past effective population size. In livestock populations, for example, recent effective population sizes are often quite small. As a result, the extent of LD can be considerable, with measurable LD (eg useable for detection of QTL) for up to tens of cM in cattle (Farnir et al, 2000), sheep (McRae et al, 2001), and pigs (Nsengimana et al, 2004). This is in sharp contrast to the situation in humans, where recent effective population sizes have been large, and consequently measurable LD often only extends a few $100 \mathrm{~kb}$ (eg Dunning et al, 2000; Reich et al, 2001). So how valid is our assumption that the past effective population size for the Atlantic salmon aquaculture breeding programmme was 100? The breeding programme for Atlantic salmon in Norway began seven generations ago, and was based on sampling fish from a number of rivers throughout Norway (Gjedrem et al, 1991). However, only a small number of the fish sampled have actually contributed to the breeding population (Gjedrem et al, 1991), and the estimated effective population size is between 200 and 50 (Mork et al, 1999). The effective population is likely to have been very much larger in the past - estimates for effective population sizes for populations from single rivers are in the order of 200 (eg Spidle et al, 2004), and the populations across rivers will be very much 
larger. This reduces the amount of LD, particularly for short chromosome segments (Hayes et al, 2003). Our simulation with larger effective population size (1000) in the past followed by a contraction in recent effective population size (to 100) was designed to reflect this situation. The results indicated that the level of LD was still sufficient to contribute to increasing the power of detection and accuracy of positioning the QTL. If estimates of historical effective population size turn out to be very much larger than 1000, a much denser marker map than we have simulated here will be required to detect and correctly position QTL.

In our simulations, we have ignored the effects of both natural and artificial selection. A QTL with an effect on a quantitative trait may also have a negative pleiotropic effect on fitness (Cabellero and Keightley, 1994). If this were the case, the frequency of the QTL allele with a favourable effect on the quantitative trait, but a negative effect of fitness, could be reduced, hence reducing the power of QTL detection. However, Hayes and Goddard (2003) still found detectable QTL segregating in populations where QTL affecting an economic trait had a pleiotropic effect on fitness and natural selection was simulated.

In our study, we have used the LDLA method proposed by Meuwissen et al (2002) to detect and position QTL. This method uses multi-locus information from both linkage and LD information simultaneously to construct a matrix of IBD coefficients, which is then fitted as the (co)variance matrix of the QTL in a variance component analysis. An alternative approach, described by Fan et al (2005), models the LD component as fixed effect mean coefficients. The Fan approach has the advantage that contribution from LA and LA can be clearly separated. However, the method of Meuwissen et al (2002) has two advantages. The first is that both LD and LA have to contribute simultaneously to the test statistic, reducing the chance of false positive results (see Meuwissen et al, 2002 for details; Gautier et al, 2006; Olsen et al, 2005). The second advantage is that treating the founder haplotypes as random effects allows a (co)variance matrix to be created between and among the founder haplotypes based on probabilities of identical by state, recombination distances between the markers, and population models of LD (Meuwissen and Goddard 2001). Given that both the method of Fan et al (2005) and Meuwissen et al (2002) have advantages and disadvantages, future work should compare the power of these two methods to detect QTL.

A substantial increase in the power of the mapping experiment is possible if the mapping population (from the breeding population) is chosen to maximise the chance that QTL are segregating in the selected families. The MAXV strategy, for example, selected families with maximum within family variance for the trait being mapped. This strategy is similar to the strategy proposed by Stella and Boettcher (2004), where the half-sib families with the most discordant sibs are selected for genotyping. They showed that for continuous phenotypes, this strategy was as powerful or more powerful in some cases than standard selective genotyping, where the most extreme sibs within a number of families are genotyped. Provided phenotypes are available before genotyping, the MAXV strategy should be used to select families for genotyping from the general population.
Our results suggest that the MAXV strategy, in combination with LDLA mapping, enables detection of QTL of moderate effect for economically important traits using the currently available commercial Atlantic salmon populations, and within the existing phenotype recording structure, without the need to create special resource populations. An advantage of detecting QTL in the commercial population is that MAS can be applied immediately, by genotyping the potential broodstock for the haplotype of markers surrounding the QTL.

\section{References}

Bell PA, Chaturvedi S, Gelfand CA, Huang CY, Kochersperger M, Kopla R et al (2002). SNPstream UHT: ultra-high throughput SNP genotyping for pharmacogenomics and drug discovery. Biotechniques 1(Suppl): 70-77.

Blott S, Kim JJ, Moisio S, Schmidt-Kuntzel A, Cornet A, Berzi P et al (2003). Molecular dissection of a quantitative trait locus: a phenylalanine-to-tyrosine substitution in the transmembrane domain of the bovine growth hormone receptor is associated with a major effect on milk yield and composition. Genetics 163: 253-266.

Cabellero A, Keightley PD (1994). A pleiotropic nonadditive model of variation in quantitative traits. Genetics 138: 883-900.

Fan R, Spinka C, Jin L, Jung J (2005). Pedigree linkage disequilibrium mapping of quantitative trait loci. Eur J Hum Genet 13: 216-231.

Farnir F, Coppieters W, Arranz JJ, Berzi P, Cambisano N, Grisart $B$ et al (2000). Extensive genome-wide linkage disequilibrium in cattle. Genome Res 10: 220-227.

Gautier M, Barcelona RR, Fritz S, Grohs C, Druet T, Boichard D et al (2006). Fine mapping and physical characterization of two linked QTLs affecting milk fat yield in dairy cattle on BTA26. Genetics 172: 425-436.

George AW, Visscher PM, Haley CS (2000). Mapping quantitative trait loci in complex pedigrees: a two-step variance component approach. Genetics 156: 2081-2092.

Gilmour AR, Gogel BJ, Cullis BR, Welham SJ, Thompson R (2002). ASReml user guide release 1.0. VSN International Ltd Hemel Hempstead HP11ES, UK.

Gjedrem T (1997). Selective breeding to improve aquaculture production. World Aquaculture 28: 33-45.

Gjedrem T, Gjøen HM, Gjerde B (1991). Genetic origin of Norwegian farmed Atlantic salmon. Aquaculture 98: 41-50.

Goddard ME (1991). Mapping genes for quantitative traits using linkage disequilibrium. Genet Sel Evol 23(Suppl 1): $131 \mathrm{~s}-134 \mathrm{~s}$.

Haley CS, Knott SA (1992). A simple regression method for mapping quantitative trait loci in line crosses using flanking markers. Heredity 69: 315-324.

Hayes BJ, Goddard ME (2003). Evaluation of marker assisted selection in pig enterprises. Livest Prod Sci 81: 197-211.

Hayes BJ, Visscher PE, McPartlan H, Goddard ME (2003). A novel multi-locus measure of linkage disequilibrium and it use to estimate past effective population size. Genome Res 13: 635-643.

Hayes B, Lærdahl J, Lien S, Berg P, Davidson W, Koop B et al (2004). Detection of single nucleotide polymorphisms (SNPs) from atlantic salmon expressed sequence tags (ESTs). Proc Euro Assoc Anim Prod Bled Slovenia.

He C, Chen L, Simmons M, Li P, Kim S, Liu ZU (2003). Putative SNP discovery in interspecific hybrids of catfish by comparative EST analysis. Anim Genet 34: 445-448.

Heath SC, Snow GL, Thompson EA, Tseng C, Wijsman EM (1997). MCMC segregation and linkage analysis. Genet Epidemiol 14: 1011-1015. 
Hudson RR (1985). The sampling distribution of linkage disequilibrium under an infinite allele model without selection. Genetics 109: 611-631.

Jackson TR, Ferguson MM, Danzmann RG, Fishback AG, Ihssen PE, O'Connell $\mathrm{M}$ et al (1998). Identification of two QTL influencing upper temperature tolerance in three rainbow trout (Onchorhynchus mykiss) half-sib families. Heredity $\mathbf{8 0}$ 143-151.

Kimura M, Crow JF (1964). The number of alleles that can be maintained in a finite population. Genetics 49: 725-738.

Lee SH, van der Werf JHJ (2004). The efficiency of designs for fine-mapping of quantiative trait loci using combined linkage disequilibrium and linkage. Genet Sel Evol 36: 145-161.

Martinez VA, Hill WG, Knott SA (2002). On the use of double haploids for detecting QTL in outbred populations. Heredity 88: 423-431.

McRae AF, McEwan JC, Dodds KG, Wilson T, Crawford AM, Slate J (2001). Linkage disequilibrium in domestic sheep. Genetics 160: 1113-1122.

Meuwissen THE, Goddard ME (1996). The use of marker haplotypes in animal breeding schemes. Gen Sel Evol 28: 161-176.

Meuwissen THE, Goddard ME (2001). Prediction of identity by descent probabilities from marker-haplotypes. Gen Sel Evol 33: 605-634.

Meuwissen THE, Karlsen A, Lien S, Olsaker I, Goddard ME (2002). Fine mapping of a quantitative trait locus for twinning rate using combined linkage and linkage disequilibrium mapping. Genetics 161: 373-379.

Moen T, Fjalestad KT, Munck H, Gomez-Raya L (2004a). A multistage testing strategy for detection of quantitative trait loci affecting disease resistance in Atlantic salmon. Genetics 167: $851-858$

Moen T, Hoyheim B, Munck H, Gomez-Raya L (2004b). A linkage map of Atlantic salmon (Salmo salar) reveals an uncommonly large difference in recombination rate between the sexes. Anim Genet 35: 81-92.

Mork J, Bentsen H, Hindar K, Skaala Ø (1999). Genetiske interaksjoner mellom oppdrettslaks og vill laks. In: Rieber-Mohn GFF (ed) Til laksat alle kan ingengjera? Omarsaker til nedgangen $i$ de norske villaksbestandene og forslag tilstrategier og tiltak fora bedre situasjonen. Norges Offentlige Utredninger. Statens forvaltningst-jeneste: Oslo. Vol 9. pp 181-200 (in Norwegian).

Nsengimana J, Baret P, Haley CS, Visscher PM (2004). Linkage disequilibrium in the domesticated pig. Genetics 166: 13951404.

Olsen H, Lien S, Gautier M, Nilsen H, Roseth A, Berg PR et al (2005). Mapping of a milk production quantitative trait locus to a $420-\mathrm{kb}$ region on bovine chromosome 6. Genetics 169: 275-283.

Perry GM, Danzmann RG, Ferguson MM, Gibson JP (2001). Quantitative trait loci for upper thermal tolerance in outbred strains of rainbow trout (Oncorhynchus mykiss). Heredity 86: 333-341.

Reich DE, Gargill M, Bolk S, Ireland J, Sabeti PC (2001). Linkage disequilibrium in the human genome. Nature 411: 199-204.

Reid DP, Szanto A, Glebe B, Danzmann RG, Ferguson MM (2005). QTL for body weight and condition factor in Atlantic salmon (Salmo salar): comparative analysis with rainbow trout (Oncorhynchus mykiss) and Arctic charr (Salvelinus alpinus). Heredity 94: 166-172.

Sakamoto T, Danzmann RG, Okamoto N, Ferguson MM, Ihssen PE (1999). Linkage analysis of quantitative trait loci associated with spawning time in rainbow trout (Oncorhynchus mykiss). Aquaculture 173: 33-43.

Sebastiani P, Lazarus R, Weiss ST, Kunkel LM, Kohane IS, Ramoni MF (2003). Minimal haplotype tagging. Proc Natl Acad Sci USA 100: 9900-9905.

Spidle AP, King TL, Letcher BH (2004). Comparison of genetic diversity in the recently founded Connecticut River Atlantic salmon population to that of its primary donor stock, Maine's Penobscot River. Aquaculture 236: 253-265.

Stella A, Boettcher PJ (2004). Optimal designs for linkage disequilibrium mapping and candidate gene association tests in livestock populations. Genetics 166: 341-350.

Sved JA (1971). Linkage disequilibrium and homozygosity of chromosome segments in finite populations. Theor Popul Biol 2: 125-141.

Withler RE, Supernault KJ, Miller KM (2005). Genetic variation within and among domesticated Atlantic salmon broodstocks in British Columbia, Canada. Anim Genet 36: 43-50. 